\title{
El desafío de la interculturalidad para la cooperación universitaria ${ }^{1}$
}

\author{
The intercultural challenge
}

\begin{abstract}
Javier Herrán Gómez, sdb²
jherran@ups.edu.ec

\section{Resumen}

Este trabajo analiza los alcances y potencialidades del convenio de cooperación académica entre la Universidad Politécnica Salesiana (UPS) y la Universidad de Viena, a la luz de los desafíos comunes de la interculturalidad, un punto pendiente tanto para Europa - de cara a las diferencias al interno de cada nación y a la población migrante- como para Latinoamérica -atravesada por la diversidad lingüística y cultural de sus pueblos.

El artículo concluye ratificando que la interculturalidad es un desafío pendiente para la educación superior en general, que puede inspirar alianzas estratégicas interuniversitarias. El potencial de tal tarea no toma en cuenta solo a los posibles beneficiarios, sino también comporta la transformación de los marcos de educación superior.
\end{abstract}

\section{Palabras claves}

Interculturalidad, educación superior, formación docente.

\begin{abstract}
This work analyses the scope and potentialities of the agreement with the academic cooperation between Universidad Politecnica Salesiana (UPS) and Viena University, in light of the common challenges of inter-culturality, a common issue pending to be addressed by Europe - in face of the differences within each nation and the migrant population — as well as by Latin American — crossed by the linguistic and cultural diversity of its nationalities.

The article concludes by ratifying that inter-culturality is a pending challenge for higher-level education in general, that can inspire strategic alliances across universities. The potential of this task does not just take into account the possible beneficiaries, but also includes the transformation of the frameworks for higherlevel education.
\end{abstract}

\section{Keywords}

Inter-culturality, higher-level education, teachers'education

Forma sugerida de citar: HERRÁN GÓMEZ, Javier, sdb (2013). "El desafío de la interculturalidad". En: Universitas, XI (19), julio-diciembre, p. 285-298. Quito: Editorial Abya Yala/ Universidad Politécnica Salesiana.

1 Ponencia presentada en la Universidad de Viena, en el marco del $54^{\circ}$ Congreso Internacional de Americanistas (15-20 de julio de 2012). Para su elaboración, se ha tomado en cuenta la información proporcionada por los siguientes docentes de la Universidad Politécnica Salesiana que llevaron adelante las actividades previstas en el convenio interinstitucional entre la Universidad de Viena y la Universidad Politécnica Salesiana. Víctor Hugo Torres (director de la Maestría en Desarrollo y Movimientos Sociales), Sebastián Granda (director de la Carrera de Educación Intercultural Bilingüe), Lola Vázquez (directora de la Carrera de Gestión para el Desarrollo Local y Sostenible), Alexandra Martínez (directora de la Maestría de Antropología) y José Juncosa (director de la Carrera de Antropología y de la editorial Abya-Yala).

2 Pedagogo, Máster en Planificación del Desarrollo, Rector de la Universidad Politécnica Salesiana. 


\section{Introducción}

Desde su creación en 1994, una de las preocupaciones centrales de la UPS ha sido entablar relaciones de trabajo e intercambio académico con instituciones de educación superior de carácter nacional e internacional, con el objetivo de cualificar la formación a nivel de pregrado y posgrado, e impulsar los procesos de investigación generativa. En este marco, la UPS ha firmado convenios académicos con diferentes universidades de la región y el mundo: Universidad de San Simón, CIESAS, Universidad Católica del Perú, Universidad Complutense de Madrid, Universidad de Ferrara, entre otras. En julio de 2011, la UPS firmó un convenio de cooperación académica con la Universidad de Viena, con la finalidad de impulsar iniciativas en el área de las ciencias sociales y de la antropología en particular.

Los compromisos adquiridos quedaron establecidos de la siguiente manera: 1) invitarse mutuamente a participar en seminarios y conferencias organizadas por las respectivas instituciones; 2) apoyar visitas mutuas de docentes e investigadores de las dos instituciones; 3 ) intercambiar estudiantes graduados y/o no graduados; 4) explorar la colaboración en educación; 5) intercambiar información científica en forma de publicaciones, material de instrucción y otros resultados de enseñanza e investigación; 6) editar conjuntamente investigaciones y textos producidos por ambas instituciones.

El convenio entre ambas instituciones, de hecho, ha permitido concretar iniciativas de intercambio docente y organización conjunta de conversatorios y cursos abiertos, cuyos resultados y actividades se ponen a consideración. La Universidad de Viena ha aportado con la experticia y trayectoria de sus docentes en tres áreas de conocimiento y aplicación de interés mutuo: 1) movimientos sociales y pueblos indígenas; 2) derechos humanos en el contexto del neoconstitucionalismo latinoamericano; 3 ) aportes teóricos y metodológicos relacionados con la antropología urbana y la antropología del consumo. La UPS, por su lado, ha puesto en consideración su recorrido respecto a la interculturalidad en el contexto de la educación superior en tres de sus ofertas carreras académicas: Pedagogía Intercultural Bilingüe, Gestión para el Desarrollo Local Sostenible y Antropología Aplicada. Además, ofrece la difusión editorial de aportes conjuntos a través de la Editorial Universitaria Abya-Yala. 
A continuación, nos proponemos reflexionar el camino recorrido conjuntamente por ambas instituciones, optando por un tono marcadamente narrativo que permite visualizar los alcances y hacer explícitos los principios que animaron las acciones.

\section{El camino recorrido}

En el marco de este convenio, en el mes de julio de 2012, se realizó un taller de trabajo en la UPS-Quito, con la participación de George Grünberg, representante de la Universidad de Viena y de la Facultad de Ciencias Sociales, y los directores de los programas académicos y centros de investigación afines al área de ciencias sociales de la UPS, para intercambiar ideas sobre los posibles ámbitos de interés y las actividades a desarrollarse. Como resultado del taller se logró consensuar un plan de trabajo que, definitivamente, contribuyó a concretar el convenio. En el plan se definieron seis espacios de trabajo que tienen ver con la docencia y la investigación, pero también con las publicaciones. A continuación presentamos los compromisos asumidos por ambas instituciones en cada uno de los ámbitos (véase el plan de trabajo a desarrollarse entre la UPS y la Universidad de Viena, en el marco del convenio académico a firmarse entre ambas instituciones, 1 de julio de 2011).

1. Apoyo a la docencia. La Universidad de Viena se compromete a apoyar a la UPS con docentes para el desarrollo de cursos de pregrado y posgrado, así como cursos de formación continua. Los docentes podrán ser estudiantes que están culminando sus estudios de doctorado, pero también docentes de la Facultad de Ciencias Sociales.

2. Pasantías y supervisión de trabajo de campo. La UPS se compromete a supervisar el trabajo de campo de los estudiantes de la Universidad de Viena que tengan interés en realizar sus tesis en el Ecuador, y a abrir sus puertas para que los estudiantes tesistas tomen materias en los programas de posgrado.

3. Colaboración en investigación. La Universidad de Viena se compromete a servir de enlace para que la UPS participe en las convocatorias del sistema europeo de fomento a la investigación de universidades euro- 
peas y latinoamericanas, y pueda integrarse a las redes de investigación sobre temáticas de interés para ambas universidades. Este ámbito incluye la posibilidad de trabajar en proyectos de investigación conjuntos.

4. Apoyo para la plataforma web. La Universidad de Viena se compromete a colaborar en programas de formación en modalidad virtual que lleva adelante la UPS, con el diseño de textos interactivos y videoconferencias. El manejo y la administración de la plataforma serán llevados a cabo por la UPS. A mediano plazo, se sugiere pensar en la posibilidad de diseñar y desarrollar, entre ambas universidades, programas de formación en modalidad virtual para ser ofrecidos en ambos países.

5. Publicación de tesis. La UPS, a través de la editorial Abya-Yala, se compromete a publicar tesis sobre América Latina de los estudiantes de maestría y doctorado en antropología de la Universidad de Viena.

6. Participación en las revistas de la UPS. Los docentes de la Facultad de Ciencias Sociales de la Universidad de Viena participarán en las revistas Universitas y Alteridad de la UPS, con artículos de su autoría. Para ello, los directores de ambas revistas enviarán, por cada número, una invitación formal a la Universidad de Viena.

Luego de casi un año de la firma del convenio, podemos sostener que los avances se han dado fundamentalmente en el ámbito de apoyo a la docencia. En febrero de 2012, la profesora María Dabringer inició su estancia en la Universidad Politécnica Salesiana de Quito y tuvo a su cargo dos seminarios: Antropología Urbana (con estudiantes de pregrado) y Antropología del Consumo (con estudiantes de posgrado). En marzo del mismo año recibimos la visita del profesor René Kuppé, quien tuvo bajo su responsabilidad dos actividades académicas: el conversatorio sobre "Neoconstitucionalismo e interculturalidad en América Latina" con los docentes de las áreas de ciencias sociales y humanidades y la conferencia abierta sobre "Los fundamentos jurídicos del Estado plurinacional" con estudiantes de pregrado de la sede de Quito.

En el ámbito de la publicación de tesis se ha avanzado en la revisión inicial de la producción sobre América Latina generada por los estudiantes de la Maestría en Antropología y en la definición preliminar de las condiciones y procedimientos para su publicación. De igual manera, respecto a la participación en las revistas, dos docentes de la Universidad de Viena han enviado artículos para 
la revista Universitas. Hacia delante, hay varios retos para las instituciones, entre los que se destacan: 1) fortalecer el intercambio de docentes a través de la visita continua de docentes de la Universidad de Viena, pero también a través de la implementación de estancias académicas de docentes de la UPS en la universidad; 2) diseñar y ejecutar programas en modalidad virtual entre las dos universidades, en áreas temáticas de mutuo interés y para estudiantes de la UPS y la Universidad de Viena; 3) implementar espacios de diálogo para definir una agenda de investigación mínima y viable entre ambas universidades.

\section{Las actividades realizadas y su aporte a la interculturalidad}

El seminario Antropología Urbana tuvo lugar del 13 al 17 de febrero de 2012 y se enfocó en los saberes básicos sobre la subdisciplina (historia, posiciones y corrientes teóricas), y en algunos de los métodos cualitativos más importantes que implica el trabajo de campo etnográfico en contextos urbanos. Participaron en el seminario estudiantes provenientes de diversas carreras, principalmente de Gerencia y Liderazgo, Psicología Social y Antropología Aplicada, casi todos jóvenes y adultos urbanos. La docente, María Dabringer, se basó en la metodología de taller mediante ejercicios grupales participativos en torno a la temática, utilizando materiales visuales y textos de apoyo, procurando acercar a los estudiantes a perspectivas antropológicas sobre la vida social en las ciudades. Asimismo, incluyó la tutoría individual en el marco del acompañamiento a los estudiantes en la realización de su respectivo trabajo de campo.

Los temas desarrollados fueron: 1) los antropólogos y las ciudades; 2) temas, perspectivas y metodologías de la antropología urbana; 3 ) el Mental Mapping como método de la antropología urbana; 4) las diferencias sociales en las ciudades y 5) la vida urbana como fenómeno identificante. Su aporte consistió en la toma de conciencia de que la diversidad atraviesa todas las subjetividades sociales y todos los espacios de la vida sociocultural. Se trata de una realidad que nos confronta a todos sin excepción con nuestros propios tejidos identitarios y llama la atención sobre el hecho de que la identidad no es una realidad reservada a las subjetividades étnicas. 
En el seminario Antropología del Consumo, desarrollado también por la docente María Dabringer, participaron 17 estudiantes de la Unidad de Posgrados de la UPS y se constituyó en una oportunidad para promover en los estudiantes de maestría un pensamiento y una práctica intercultural. La modalidad del seminario fue semipresencial e involucró dos encuentros presenciales de dos fines de semana completos, así como tutorías presenciales, observaciones etnográficas sobre actos de consumo y producción de trabajos finales a través de la plataforma virtual. Los principales participantes fueron los estudiantes de la Maestría de Antropología y de la Maestría en Políticas Sociales y, como es usual en nuestra universidad, pertenecen a distintos pueblos y nacionalidades, clases sociales y estratos económicos.

El objetivo de este seminario fue lograr que los estudiantes se aproximaran a la comprensión del consumo no solo como fenómeno social, cultural y económico influido local y globalmente sino también como constructor de identidades. Para ello se desarrollaron dos temas específicos: 1) el consumo como señalador de diferencias culturales, de género y de clase, y 2) la relación del consumo urbano con los estilos de vida. En el primer caso, se abordó la dimensión social y cultural asumiendo la diferencia cultural como elemento clave del consumo en tanto práctica que estructura la vida cotidiana y las identidades. Para el segundo caso, el análisis del consumo urbano y de los estilos de vida, se discutió y problematizó la relación entre procesos de homogenización del consumo y la importancia de lo "tradicional" y lo "auténtico" partiendo del enfoque que propone García Canclini (2006: 83) según el cual el consumo es un lugar de diferenciación social, distinción simbólica y un proceso ritual que implica desarrollar un pensamiento crítico respecto a la diversidad y a las prácticas de distinción generadas por actores de distintas clases sociales, grupos étnicos y géneros.

Durante todo el seminario, la profesora Dabringer enfatizó la metodología participativa lo cual facilitó el intercambio de ideas y experiencias entre estudiantes y entre estudiantes y profesora. Además se utilizaron materiales visuales y ciertos apoyos pedagógicos como artículos de periódicos, anuncios publicitarios, videos sobre producción, procesamiento y consumo de alimentos en África, América del Sur y Europa. En definitiva, se procuró que los estudiantes pudieran entender cómo ciertas prácticas de consumo locales se transforman en 
prácticas globales y viceversa. Esta metodología desencadenó fructíferas discusiones que pusieron en el tapete la tensión que existe en América Latina entre una "estructura nacional históricamente consolidada y las políticas modernizadoras" que interpelan a la aparentemente ganada integración y comunicación entre clases y etnias en América Latina (García Canclini, 2006: 96) a través del mestizaje o la cultura criolla (Portocarrero, 2004: 2-3).

En términos pedagógicos, son dos los logros obtenidos. Por un lado, los estudiantes se involucraron como antropólogos y antropólogas en el estudio del consumo en distintos ámbitos: alimentación, productos culturales, trabajo sexual, servicios médicos, y desde allí pudieron aproximarse a mirar el consumo como producto cultural, económico y social. Segundo, el curso permitió que los estudiantes pudieran pensar el consumo como un doble proceso de homogenización y localización de productos (Dabringer, 2011) que permite entender la influencia de determinadas prácticas de mercado en las culturas locales, en las identidades étnicas, de género y de clase.

Si entendemos la interculturalidad desde el enfoquede Tubino (2004), como una manera de comportarse, una propuesta ética, una forma de ser y, además, como un concepto en construcción que debe ser teorizado, el curso de Antropología del Consumo fue una oportunidad para promover en los estudiantes de maestría un pensamiento y una práctica intercultural. En efecto, los estudiantes a partir de sus lecturas, trabajos etnográficos e interacción con la profesora tuvieron la oportunidad de desarrollar pensamiento crítico respecto al consumo como un fenómeno social y cultural, diverso y, contradictoriamente, cada vez más homogeneizado. Además, pudimos constatar que para entender el consumo es necesario un acercamiento no solamente desde la tolerancia (Portocarrero, 2004) sino desde la afirmación positiva de las identidades que sustentan estas diversidades y sobre todo, es imprescindible lograr cambios en las prácticas y en la ética de las personas y naciones (Martinez, 2007: 15).

El tercer aporte ha sido realizado por René Kuppe, de la Universidad de Viena, sobre el nuevo constitucionalismo que experimentan algunos países de la región en el marco de los conversatorios con académicos y estudiantes organizado por la Cátedra Monseñor Proaño de nuestra universidad. Kuppe abordó los siguientes temas: la dimensión pluriétnica del nuevo constitucionalismo latinoamericano; los derechos amparados en la Constitución ecuatoriana de 
2008; la jurisdicción indígena; la consulta previa y los derechos de los pueblos indígenas. Señaló que los debates sobre los derechos indígenas impactaron las reformas constitucionales en muchos países de América Latina, especialmente durante las décadas de los 80 y 90 del siglo XX. Sin embargo, esta primera ola de reformas no respondió plenamente a las demandas indígenas pues tuvo lugar en el marco de las reformas "liberales" cuyo objetivo es el fortalecimiento de un "Estado de derecho". Paradójicamente, se reconocía el derecho, pero el control y los mecanismos para su implementación se alojaron en las cortes u otras instituciones "occidentales" no indígenas. Frente a este modelo del "Estado de derecho liberal", los movimientos indígenas implementan un nuevo activismo constitucional (que incluye también a otros sectores de la sociedad) cuya expresión más importante es el surgimiento del término "plurinacional" que implica confrontar las bases mismas del Estado de derecho liberal y cuestiona el sistema normativo controlado y definido por el Estado. Plantea, a cambio, el desarrollo de nuevas instituciones y una nueva relación entre Estado y sociedad. El discurso y la lucha indígena es, en suma, una contribución a la descolonización y a la democratización del derecho.

El enfoque liberal de ciertas Constituciones reconoce al individuo como sujeto de Derecho y garantiza la ciudadanía y la igualdad ante la ley en forma individual. El constitucionalismo (o neoconstitucionalismo) plurinacional plantea al sujeto colectivo como el centro de derechos y establece la primacía de los derechos sobre el poder. Por eso Ecuador, en su Constitución, se proclama como "Estado Nacional de Derechos".

La Constitución del Ecuador, además, debate dos ideas fundamentales: el régimen de desarrollo y el régimen del "buen vivir". El "régimen de desarro1lo", como lo entiende Kuppé, es el conjunto organizado, sostenible y dinámico de los sistemas económicos, políticos, socioculturales y ambientalistas que garantizan la realización del buen vivir. El "régimen del buen vivir", en cambio, apunta a que las personas, comunidades y pueblos gocen efectivamente de todos sus derechos y ejerzan responsabilidades en el marco de la interculturalidad y la convivencia con la naturaleza. Todos estos temas, recogidos en la Constitución, constituyen un gran avance no solamente en términos de la normativa jurídica, sino también para la conquista de los derechos sociales. Sin embargo, alerta que el reconocimiento no necesariamente significa su total aplicación. 


\section{La Universidad Politécnica Salesiana y la interculturalidad}

La interculturalidad es una opción prioritaria de la UPS que se expresa en su oferta académica, en las prioridades y líneas investigativas y en los convenios de vinculación con la comunidad. En 1994, la UPS inició el Programa Académico Cotopaxi, del cual se constituiría, luego, la Carrera de Educación Intercultural Bilingüe, hoy por hoy, la iniciativa de formación profesional para profesores indígenas de enseñanza básica más importante en el Ecuador, con centros de apoyo en seis provincias. Hasta el momento, ha graduado a más de 300 profesores indígenas, casi todos ellos pertenecientes al pueblo Kichwa, pero también de las nacionalidades indígenas Shuar y Achuar, de la Amazonía (véase Farfán, 2009, 2006; Granda e Iza, 2012). La Carrera de Gestión del Desarrollo Local y Sostenible, creada en 1995, en cambio, se orienta a la formación de líderes comunitarios, autoridades locales y a la dirigencia de las organizaciones indígenas, afroecuatorianas y populares. Entre los ejes que articulan su propuesta constan las políticas públicas interculturales, las herramientas participativas de gestión comunitaria, la soberanía alimentaria y el buen vivir. Finalmente, la Carrera de Antropología Aplicada (la más antigua de las tres porque inició en 1987) apunta a la profesionalización de personas insertas en comunidades diversas como misioneros, educadores, animadores de organizaciones y gestores de la cultura. Su principal objetivo consiste en formar profesionales antropólogos capaces de fomentar la interculturalidad en múltiples espacios de la vida sociocultural y el buen vivir: la salud, el desarrollo, las políticas públicas, el patrimonio, el fortalecimiento organizacional (véase Bartoli, 2002; Andrade, 2011; Cucurella y Vallejo, 2012).

A estas ofertas debemos añadir otras de posgrado, como las maestrías de Antropología y en Desarrollo Local con mención en Formulación de Proyectos de Desarrollo Endógeno y en Desarrollo Local con mención en Movimientos Sociales, cada una de las cuales ha graduado un número significativo de líderes indígenas y afroecuatorianos, y cuyos trabajos de grado ponen en escena los nudos problemáticos de la interculturalidad en diversos aspectos de la vida sociocultural.

En efecto, las propuestas académicas atravesadas por la interculturalidad se caracterizan por la modalidad semipresencial de estudios, es decir, el lugar de 
aprendizaje es, al mismo tiempo, el lugar de trabajo y no supone el desarraigo de los estudiantes de sus espacios de inserción y pertenencia. Los contenidos, por tanto, se consideran herramientas de interpretación y reorientación de la práctica cotidiana no tanto de los individuos sino de los sujetos colectivos que los identifican. Ello implica, a su vez, que el perfil deseable de los estudiantes corresponda a un adulto dotado de una muy especial capacidad de autonomía para el manejo de los contenidos y la constitución de sus propios objetos de conocimiento en función de las opciones éticas y políticas que los marcan.

El segundo frente en el que la UPS profundiza su opción por la interculturalidad es la investigación, impulsada por las actividades de vinculación con la comunidad de las carreras de pregrado y posgrado. Para ello, la UPS ha creado, en 2007, el Centro de Estudio Interculturales (CEI), cuyas líneas de investigación toman en cuenta las opciones institucionales pero también las orientaciones del Plan Nacional del Buen Vivir, en lo referente a la construcción de un Estado plurinacional e intercultural. Los ámbitos en los que el CEI debe impulsar la interculturalidad son los siguientes: Estado, ciudadanía y política públicas; desarrollo, territorio y medio ambiente; derechos lingüísticos; epistemologías y diálogo de saberes; espiritualidades y teologías; comunicación; salud y finalmente educación (CEI, 2011). El CEI, además de desarrollar varios proyectos en convenio con instituciones gubernamentales y no gubernamentales, acaba de diseñar un programa de investigación trianual con los siguientes proyectos, cuyos responsables son docentes doctorandos de la UPS (CEI, 2012).

Tabla 1

Programa de estudios interculturales 2012-2014 (CEI-UPS)

\begin{tabular}{|l|l|}
\hline \multicolumn{1}{|c|}{ Título del proyecto } & Docente investigador \\
\hline Movimiento indígena y Estado & Sebastián Granda \\
\hline Las fronteras del Estado en el Ecuador contemporáneo & Víctor Hugo Torres \\
\hline Epistemologías indígenas y educación superior & José Juncosa \\
\hline Interculturalidad y teologías de la liberación en el Ecuador & Rubén Bravo \\
\hline $\begin{array}{l}\text { Sabidurías andinas y cimarronas: Aportes teóricos, metodológicos y } \\
\text { espirituales a la interculturalidad }\end{array}$ & Patricio Guerrero \\
\hline
\end{tabular}

Fuente: CEI, 2012 
La vinculación con la comunidad se ha constituido en un escenario sumamente importante cuyas actividades y convenios expresan nuestra opción por la interculturalidad mediante la atención a diversas demandas, provenientes tanto de los sectores públicos como privados, comunitarios o académicos. Por ejemplo, la Unidad de Vinculación con la Comunidad ha impulsado, desde el CEI, las siguientes investigaciones relacionadas con la situación de los pueblos y nacionalidades indígenas y afroecuatorianas: 1) indicadores sociodemográficos de las catorce nacionalidades indígenas del Ecuador, para la Subsecretaría de Pueblos, Participación y Movimientos Sociales; 2) caracterización de la oferta educativa en educación técnica vocacional y percepciones sobre las nociones de desarrollo de los pueblos y nacionalidades indígenas de la Amazonía del Ecuador para UNICEF, en el contexto de un proyecto regional y continental.

La participación en redes de conocimiento vinculadas con la interculturalidad se expresa en animar la participación de los docentes en congresos e investigaciones de alcance latinoamericanos. Por ejemplo, del 17 al 19 de noviembre de 2011 se realizó en la sede Quito el segundo seminario-taller del proyecto de investigación regional "Plurinacionalidad, ciudadanía y educación superior en América Latina". El seminario tuvo como objetivo central socializar los resultados de la investigación-acción ejecutados en cada uno de los países participantes sobre los avances en materia de plurinacionalidad y autonomía. En el encuentro participaron 16 delegados de universidades convencionales e indígenas de México, Colombia, Bolivia y Ecuador. Por Ecuador participaron la Universidad Politécnica Salesiana y la Universidad Intercultural Amawtay Wasi. Como invitados especiales estuvieron Luis Maldonado -líder quichua ecuatoriano- y Ricardo Cuenca del Instituto de Estudios Peruanos. La segunda fase del encuentro tuvo lugar en San Cristóbal, México, en abril de 2012, con el propósito de presentar los resultados finales del proyecto y acordar criterios para la publicación de las investigaciones.

Asimismo, desde la vinculación con la comunidad favorecemos la participación activa de nuestros docentes y estudiantes en encuentros y simposios como: 1) Congreso Voces e imágenes de las Lenguas en Peligro (del 7 al 11 de septiembre de 2011) coorganizado con la PUCE y en el cual la UPS tuvo a su cargo la realización del simposio sobre derechos lingüísticos; 2) la coorganización del encuentro binacional de organizaciones afroamericanas "Al otro la'o 
de la raya", con el Ministerio de Relaciones Exteriores del Ecuador y la Organización Interamericana de Educación (12 y 13 de diciembre de 2011).

Para finalizar este apartado, quiero mencionar un último escenario de desarrollo de la interculturalidad y que corresponde al ámbito editorial. Para ello, la UPS ha realizado un convenio de cooperación académica con la editorial Abya-Yala, creada por el padre Juan Bottasso -que posee un historial de 37 años ininterrumpidos al servicio de la difusión de la producción académica sobre los pueblos diversos. Esta alianza estratégica contribuirá a que la editorial radicalice su opción y, al mismo tiempo, a que la UPS incremente la producción de temas relacionados con la interculturalidad. De hecho, ha publicado muchas memorias de los Congresos de Americanistas, desde el organizado en Manchester (1982), de manera ininterrumpida hasta el presente. Destacamos que incluso algunos profesores de la Facultad de Ciencias Sociales de la Universidad de Viena han publicado sus investigaciones en Abya-Yala, por ejemplo, George Grümberg (Articulación de la diversidad. Tercera reunión de Barbados, AbyaYala, 1995) y Elke Mader (Metamorfosis del poder. Persona, mito y visión en la sociedad shuar y achuar, Abya-Yala, 1999).

Quisiéramos que la Universidad de Viena comparta con nosotros la visión de que el mundo editorial es una herramienta indispensable para conectar y generar redes de conocimiento compartido y de acción conjunta entre diverso tipo de actores: académicos, movimientos sociales, comunidades locales, activistas, difusores de la cultura, etc.

\section{Conclusiones y desafíos futuros}

Para finalizar, planteamos algunos desafíos futuros para ambas instituciones, no tanto a partir de las actividades programadas que restan por materializar, sino desde las posibilidades que plantea el horizonte siempre inconcluso de la interculturalidad. Unos desafíos que pueden expresarse a manera de ideasfuerza con implicaciones éticas, políticas y epistémicas:

1. Las actividades realizadas nos incitan a trascender el enfoque liberal sobre la diversidad expresado en la apuesta del multiculturalismo, que consiste en admitir, tolerar e incluso valorar la diversidad, pero sin generar relaciones 
equitativas entre los pueblos y sus culturas; tampoco entre sus respectivos patrones civilizatorios respecto a la vida y la naturaleza, la producción y el conocimiento, ocultando las hegemonías y relaciones de dominación que naturalizan las pautas culturales dominantes y subordinan las diversas. Para nosotros, la interculturalidad va más allá del multiculturalismo, consiste en la práctica de una relación con equidad y respeto "entre" los pueblos y las culturas.

2. Proponemos trabajar duramente por una academia intercultural, que va mucho más allá de la perspectiva de inclusión en las aulas universitarias -todavía necesaria pero incompleta- de los indígenas y los afroamericanos o de las subjetividades colectivas que se autoproponen como diversas. Pensamos que la academia, si quiere renovarse, debe partir de las formas de pensar, de las metodologías, epistemes y lenguas que ella misma excluyó y subordinó al considerarlas no científicas, atrasadas o ancestrales. Ello implica abrir la Universidad a otros actores, a dedicar energías para dar la palabra a quienes se la negamos. Solo así será posible que la Universidad sea, cada vez más, un escenario de diálogo de saberes y un espacio de equidad epistémica.

\section{Bibliografía}

Andrade, S.

2012 Informe del proyecto de investigación "La conformación y el desarrollo de la antropología como disciplina en el Ecuador". Quito: Universidad Católica del Ecuador.

Bartoli, L.

2002 Antropología Aplicada: Historia y perspectivas desde América Latina. Quito: Abya-Yala.

CEI (Centro de Estudios Interculturales)

2011 “Líneas de investigación 2011-2014”. Quito: UPS.

CEI (Centro de Estudios Interculturales)

2012 "Programa de estudios interculturales". Quito: UPS.

Cucurella, L. y Vallejo, C.

2012 “Abya Yala y el retorno de los pueblos". En: varios autores, La presencia salesiana en Ecuador. Perspectivas históricas y sociales. Quito: Abya-Yala. 
Dabringer, M.

“Consumo local/global en Quito: 'Tradiciones globalizadas' en elcontexto urbano”. En: varios autores, Culturas politicas en la Región Andina. Madrid: Vervuert/Iberoamericana.

Farfán, $\mathrm{M}$.

2009 "Experiencia del Programa Académico Cotopaxi, formación en Educación Intercultural Bilingüe de la Universidad Politécnica Salesiana”. En: Mato, D. (coord.), Diversidad cultural e interculturalidad en educación superior. Experiencias en América Latina. Caracas: UNESCO.

García Canclini, N.

2006 "El consumo cultural: una propuesta teórica". En: Guillermo (coord), El consumo en América Latina. Construcción teórica y líneas de investigación. Bogotá: Convenio Andrés Bello.

Granda, S. e Iza Remache A.

2012 "Los salesianos, la educación superior y los pueblos indígenas. El caso del Programa Académico Cotopaxi”. En: varios autores, La presencia Salesiana en Ecuador. Perspectivas históricas y sociales. Quito: Abya-Yala.

Grünberg, G. et al.

1987 Articulacion de la diversidad: pluralidad étnica, autonomías y democratización en América Latina. Quito: Abya-Yala.

Mader, E.

1999 Metamorfósis del poder. Persona, mito y visión en la sociedad shuar y achuar. Quito: Abya-Yala.

Martinez, A. y Granda, S.

2007 Curso de formación de ciudadanía intercultural en el progrgama de educación intercultural bilingüe. Lima: Fondo Editorial de la Pontificia Universidad Católica del Perú.

Portocarrero, G.

2004 "La interculturalidad es algo por hacer". En: Revista Cultural Electrónica Construyendo Nuestra Interculturalidad. $\mathrm{N}^{\mathrm{o}}$ 1, pp. 1-4.

Tubino, F.

2004 El interculturalismo latinoamericano y los Estados nacionales. México: Cuétzlan. 